\title{
Late Cardiovascular Toxicity Following Chemotherapy for Germ Cell Tumors
}

\author{
Darren R. Feldman, MDa,c; Wendy L. Schaffer, MD, PhD ${ }^{\mathrm{b}, \mathrm{c}}$; and Richard M. Steingart, MD ${ }^{\mathrm{b}, \mathrm{c}}$; \\ New York, New York
}

\begin{abstract}
The introduction of cisplatin-based chemotherapy has transformed germ cell tumors (GCTs), the most common malignancy to affect young adult men, into a highly curable cancer, even in the setting of advanced disease. However, over the past decade, the success of these chemotherapy regimens in curing GCTs has been temporized by an increasing recognition of their important late toxicities, such as cardiovascular disease. The relative risk of coronary artery disease in this population is particularly elevated within the first 10 years of follow-up, when patients are still in their 30s and $40 \mathrm{~s}$, which are age groups often considered too young to experience cardiovascular events. Two hypotheses have been proposed to explain the association between chemotherapy and cardiovascular disease in this population. The direct hypothesis asserts that chemotherapy causes diffuse endothelial damage, including in the coronary arteries, gradually leading to cardiovascular disease. In contrast, the indirect hypothesis proposes that chemotherapy leads to an increased incidence of cardiovascular disease risk factors, such as hypertension, hyperlipidemia, and the metabolic syndrome, which in turn enhance the risk of cardiovascular disease. This article summarizes the data on the association between chemotherapy (predominantly cisplatin-based) and the development of cardiovascular disease among GCT survivors, and reviews the evidence supporting both mechanistic hypotheses. In addition, recommendations are provided for the management of GCT survivors who received cisplatin-based chemotherapy and are therefore at risk for cardiovascular toxicity. (JNCCN 2012;10:537-544)
\end{abstract}

From ${ }^{a}$ Genitourinary Oncology Service, Division of Solid Tumor Oncology, Department of Medicine, and ${ }^{b}$ Cardiology Service, Department of Medicine, Memorial Sloan-Kettering Cancer Center; and 'Department of Medicine, Weil College of Medicine, Cornell University, New York, New York.

Submitted November 9, 2011; accepted for publication January 21, 2012

The authors have disclosed that they have no financial interests, arrangements, or affiliations with the manufacturers of any products discussed in this article or their competitors.

Correspondence: Darren R. Feldman, MD, Memorial SloanKettering Cancer Center, 1275 York Avenue, New York, NY 10065.

E-mail: feldmand@mskcc.org
Together, cancer and cardiac disease account for half of all deaths each year in the United States. ${ }^{1}$ Although as causes of death, these 2 illnesses are considered mutually exclusive, in life they often coexist and evidence is accumulating that they are linked. This issue is particularly relevant to cancer survivors, who represent a rapidly growing population. Today, nearly two-thirds of all patients with cancer are expected to be alive 5 years after their diagnosis, and in the United States the number of cancer survivors now exceeds 11 million. ${ }^{2}$

Germ cell tumors (GCTs), the most common tumor to affect men between ages 15 and 35 years, have long been considered a model for the curable malignancy, ${ }^{3}$ because even patients with widely metastatic disease can be cured with cisplatin-based chemotherapy with or without surgery. Unfortunately, over the past 2 decades, data have emerged linking chemotherapy, particularly cisplatin, with several late toxicities, with cardiovascular disease chief among them. Much of the evidence for this association has arisen from follow-up studies of patients with advanced GCTs, who, because of their young age at diagnosis, nearly universal treatment with cisplatin, and long life expectancy after therapy, are at particular risk for late toxicities. This article reviews the available evidence on the association between chemotherapy (predominantly cisplatinbased) and cardiovascular disease. The evidence supporting each of the 2 proposed mechanisms for this association, known as the direct and indirect vascular damage hypotheses, ${ }^{4}$ is summarized and recommendations for how to approach cisplatin-treated GCT survivors, given their potential cardiovascular risks, are provided. Additional late toxicities occurring in this population after treatment were reviewed elsewhere. ${ }^{4,5}$ 
Feldman et al.

\section{Defining the Risk}

Remarkably, within only 1 year of the initial publication $^{6}$ describing the success of cisplatin-based chemotherapy in curing testicular GCTs, a case report was published documenting unexpected obstructive coronary atherosclerosis identified on autopsy in 2 patients in their 20s who were treated for and died of advanced GCTs. ${ }^{7}$ Additional case reports of cardiovascular events ensued, but estimates of the incidence of this toxicity were not possible until recently. In 2000, Meinardi et $\mathrm{al}^{8}{ }^{8}$ reported on the cardiovascular outcomes of 87 GCT survivors, all younger than 50 years of age (median age, 41 years) at time of evaluation, who had received cisplatin-based chemotherapy at a single center in the Netherlands, had been followed for at least 10 years since completion of chemotherapy, and had never received radiation. With a median follow-up of 14 years, 5 patients (6\%) had experienced documented myocardial infarction or angina, the latter consisting of ischemia noted on stress testing and/or significant disease noted on coronary angiography. The relative risk was 7 times that of age-matched controls who had stage I testicular GCTs and had been treated with orchiectomy alone.

Huddart et al. ${ }^{9}$ identified a similar $7 \%$ incidence of angina, myocardial infarction, or sudden cardiac death among 360 patients treated with chemotherapy in the United Kingdom, with an ageadjusted relative risk of 2.6 compared with patients with GCTs treated with surgery alone. In a subsequent retrospective study of a nationwide cohort of 2339 5-year GCT survivors treated in the Netherlands between 1965 and 1995, investigators found an increased incidence of coronary heart disease, defined as myocardial infarction or angina, compared with age and sex-matched data in the general Dutch population, with a standardized incidence ratio of $1.17(95 \% \mathrm{CI}, 1.04-1.31) .{ }^{10} \mathrm{With}$ a median follow-up of 18 years, the absolute incidence of cardiac events was $6.3 \%$ among the 664 patients treated with chemotherapy alone. The calculated 20 -year actuarial risk for all patients was $10.3 \%$ for myocardial infarction or angina, and $7.2 \%$ for myocardial infarction alone. ${ }^{10}$ This risk was 2 -fold higher than the age-matched general population up to 54 years of age, and for patients of all ages within 5 to 9 years of completing treatment. ${ }^{10}$ Relative risks for older patients (age $>54$ years) and those followed for longer periods ( $\geq 10$ years) were less significant. In a follow-up study, the relative risk was also higher when subdiaphragmatic radiation was combined with chemotherapy compared with chemotherapy alone (standardized incidence ratio [SIR], 2.3; 95\% CI, 2.6-4.9 vs. SIR, 1.4; 95\% CI, 1.6-3.1). ${ }^{11}$ Together, these data, which are summarized in Table $1^{8-10,12}$ (older and smaller studies were reviewed elsewhere ${ }^{9,13}$ ), suggest premature coronary artery disease may be a significant cause of late morbidity and mortality in GCT survivors, warranting further exploration of the pathophysiology and impact of clinical interventions.

\section{Mechanisms}

\section{Chemotherapy-Induced Endothelial Dysfunction (Direct Hypothesis)}

Accumulating evidence shows that direct vascular injury plays an important role in the pathophysiology of late cardiovascular effects of chemotherapy in GCT survivors. Early on, Raynaud's phenomenon (RP) was identified in 22 of 60 (37\%) GCT survivors treated with bleomycin and vinblastine (with or without concurrent platinum-based therapy). ${ }^{14}$ Subsequent prospective observational studies estimate that the prevalence of RP in GCT survivors according to self-reporting or physician assessment ranges from $25 \%$ to $61 \% .{ }^{15-19}$ Symptoms generally begin within 4 to 12 months of chemotherapy, and although many patients experience resolution or at least improvement over time, persistent RP lasting up to 20 years has been reported in up to $25 \%$ of patients. $^{20}$

Investigations into the mechanism of chemotherapy-induced RP have also revealed abnormal vascular findings in patients with GCTs. In one study, abnormal hand arteriograms were noted with diffuse arterial narrowing and vascular cutoffs. ${ }^{14} \mathrm{~A}$ subsequent study of GCT survivors 4 to 9 years postcompletion of cisplatin, vinblastine, and bleomycin (PVB) found that although $44 \%$ of patients reported symptoms of RP, $100 \%$ showed an exaggerated vasomotor response to cold. ${ }^{21}$ Similar prolonged vasoconstriction responses to cold among patients treated with PVB have been observed in other studies. ${ }^{22} \mathrm{Ab}$ normal nailfold capillary morphology has also been identified on videomicroscopy in greater than $90 \%$ of patients with GCTs treated with vinblastine, bleomycin, adriamycin, and cisplatin. ${ }^{18}$ 
Chemotherapy-Induced Cardiovascular Toxicity

Table 1 Incidence and Relative Risk of Coronary Artery Disease in Chemotherapy-Treated Patients in Select Studies Since 2000

\begin{tabular}{|c|c|c|c|c|c|c|}
\hline Author (Year) & CAD Definition & $\mathbf{N}$ & $\begin{array}{l}\text { Dates of } \\
\text { Chemotherapy }\end{array}$ & $\begin{array}{l}\text { Median } \\
\text { Follow-Up } \\
\text { (Range) }\end{array}$ & $\begin{array}{l}\text { CAD } \\
\text { Incidence }\end{array}$ & $\begin{array}{l}\text { CAD } \\
\text { Relative } \\
\text { Risk }\end{array}$ \\
\hline $\begin{array}{l}\text { Meinardi et al. }{ }^{8} \\
(2000)\end{array}$ & $\begin{array}{l}\text { MI or angina with proven } \\
\text { myocardial ischemia }\end{array}$ & 87 & Before 1987 & $\begin{array}{l}14 \mathrm{y} \\
(10-20 \mathrm{y})\end{array}$ & $5(5.7 \%)$ & $7.1^{\mathrm{a}}$ \\
\hline $\begin{array}{l}\text { Huddart et al. }{ }^{9} \\
(2003)\end{array}$ & $\begin{array}{l}\text { MI, angina, or SCD (based } \\
\text { on medical records or GP } \\
\text { communication) }\end{array}$ & 390 & 1982-1992 & $\begin{array}{l}9.7 \mathrm{y} \\
(0-20 \mathrm{y})\end{array}$ & $26(6.7 \%)$ & $2.6^{\mathrm{b}, \mathrm{c}}$ \\
\hline $\begin{array}{l}\text { Van den Belt- } \\
\text { Dusebout et } \\
\text { al. }{ }^{10}(2006)\end{array}$ & $\begin{array}{l}\text { MI or angina (based on } \\
\text { medical records or GP } \\
\text { communication) }\end{array}$ & 664 & 1965-1995 & $\begin{array}{l}18.4 y \\
(5.0-38 y)^{d}\end{array}$ & $42(6.3 \%)$ & $1.35^{\mathrm{a}}$ \\
\hline $\begin{array}{l}\text { Haugnes et al. }{ }^{12} \\
(2010)\end{array}$ & $\begin{array}{l}\text { MI or angina ( } 81 \% \text { of } \\
\text { patients with angina had } \\
\text { cardiac revascularization) }\end{array}$ & 364 & 1980-1994 & $\begin{array}{l}19 y \\
(13-28 y)^{d}\end{array}$ & $52(5.6 \%)$ & $2.6^{\mathrm{b}}$ \\
\hline
\end{tabular}

Abbreviations: CAD, coronary artery disease; GP, general practitioner; MI, myocardial infarction; SCD, sudden cardiac death abserved/event ratio compared with general male Dutch population.

${ }^{b}$ Compared with patients with testicular cancer managed with surveillance (no chemotherapy or radiation) in the same cohort. 'Relative risk by age-adjusted logistic regression.

${ }^{d}$ Median follow-up time reflects the authors' entire study cohort, not limited to only those treated with chemotherapy.

Regarding specific causal agents, bleomycin has the strongest association with RP. In a study of 395 patients with good-risk nonseminomas randomized to receive 4 cycles of etoposide and cisplatin with (BEP) or without bleomycin (EP), $8 \%$ of patients in the BEP arm developed acute RP compared with none in the EP arm. ${ }^{23}$ Vinblastine and cisplatin have also been implicated as contributing to this toxicity. ${ }^{15-18}$ For example, higher rates of digital ischemia were identified in patients treated with cisplatin compared with those who were not (41\% vs. $21 \%) .{ }^{14}$ The cumulative dose of chemotherapy and the prevalence of conventional risk factors for peripheral arterial disease, such as hypertension or smoking, may also play a role. ${ }^{15,16}$

In vitro studies and laboratory findings further support a direct mechanism of cardiovascular disease through chemotherapy-induced endothelial dysfunction. For example, exposure of endothelial cells to cisplatin or bleomycin in vitro leads to endothelial cell cytokine release and cytotoxicity. ${ }^{24,25}$ Microalbuminuria, indicative of systemic vascular dysfunction due to generalized endothelial dysfunction, is present in an increased number of young GCT survivors. Furthermore, microalbuminuria independently predicts for vascular events, including stroke and myocardial infarction. ${ }^{26}$ In one study of GCT survivors (median age, 37 years) with a median follow-up of 7 years, microalbuminuria was present in $12 \%$ of patients treated with chemotherapy but in fewer than $1 \%$ of those treated with orchiectomy alone or healthy age-matched controls. ${ }^{20} \mathrm{With}$ a longer median follow-up of 14 years, microalbuminuria was detected in $22 \%$ of patients with GCTs treated with cisplatin-based chemotherapy despite all patients being younger than 50 years at evaluation (median age, 41 years). ${ }^{8}$

Elevations of additional laboratory markers of inflammation and endothelial dysfunction have also been detected in patients with GCTs acutely and during long-term follow-up after chemotherapy. In a study of patients with GCTs who completed cisplatin-based chemotherapy within the prior 3 months, significantly increased levels of von Willebrand factor were detected. ${ }^{27}$ With longer follow-up (median 7 years posttreatment), increased levels of fibrinogen, tissue-type plasminogen activator, and high-sensitivity C-reactive protein were also observed in chemotherapy-treated patients with GCTs compared with age-matched controls. ${ }^{20}$ Finally, a recent report showed an increase in circulating endothelial cells (from endothelial injury) among GCT survivors ( $>2$ years from diagnosis) who had received chemotherapy compared with those who were chemotherapynaïve. ${ }^{28}$ These findings suggest the acute onset and persistence of endothelial dysfunction related to chemotherapy. 
Feldman et al.

Carotid intimal medial thickness (IMT) has been found to correlate with increased risk of cerebrovascular accidents and myocardial infarctions in patients without known coronary artery disease. ${ }^{29}$ The carotid IMT increased significantly over a 3.5-month course of cisplatin-based chemotherapy in patients with GCTs compared with the annual change in IMT in the general population. ${ }^{27}$ Whether this finding is relevant to long-term GCT survivors is not yet clear, but lends further support to the hypothesis that direct vascular injury plays a role in long-term cardiac toxicity.

What is intriguing is that even in patients treated with orchiectomy alone, some markers of endothelial function seem to be abnormal relative to controls. ${ }^{20}$ This suggests that chemotherapy may not be the sole etiologic insult responsible for the early onset and increased incidence of cardiovascular disease in this population, but that multiple pathways and factors likely contribute to therapy-related vascular injury. These may include an inflammatory response with cytokine release, oxidative damage, hormonal imbalances, metabolic disturbances, changes in electrolytes, and platelet aggregation. For example, cisplatin injection into the umbilical vein causes marked elevations of interleukin (IL)-1 and IL-6, and cisplatin-induced nephrotoxicity increases tumor necrosis factor (TNF)- $\alpha$ levels, leading to upregulation of additional cytokines, such as TNF- $\beta$, IL-1 $\beta$, RANTES (regulated upon activation, normal T-cell expressed and secreted), macrophage inflammatory protein 2 (MIP-2), monocyte chemoattractant protein 1 (MCP-1), and T-cell activation gene 3 (TCA-3).$^{30}$ Cisplatin may also cause an increase in reactive oxygen species, leading to nuclear factor kappa-B secretion and a proinflammatory response that results in mitochondrial dysfunction. ${ }^{31}$ Platelet aggregation in response to cisplatin has also been proposed to explain the increased incidence of myocardial infarction and cerebrovascular thrombotic events in young patients with no other vascular disease risk factors. ${ }^{32}$ Finally, cisplatin might also induce vascular injury through causing hypomagnesemia, leading to vasospasm. ${ }^{33-35}$

\section{Induction of Cardiac Risk Factors (Indirect Hypothesis)}

Multiple studies have suggested an increased frequency of cardiovascular disease risk factors in patients treated for GCTs, supporting an alternative, more-indirect explanation for the increase in vascular events in chemotherapy treated GCT survivors. Select studies are reviewed in Table 2. 8,9,12,18,20,36-38

Hyperlipidemia has been reported in up to $80 \%$ of chemotherapy-treated GCT survivors, although a wide range has been observed (32\%-82\%), with methodology and definitions varying by study. ${ }^{4,8}$ In a study of 62 patients treated with chemotherapy who, at evaluation, were younger than 50 years and at a median of 14 years posttreatment, 79\% had elevated cholesterol, 35\% had low high-density lipoprotein (HDL), and 25\% had elevated triglycerides. All of these values were significantly abnormal compared with controls treated with orchiectomy alone. ${ }^{8}$ An observational study of 32 chemotherapy-treated survivors who were a median of 15 years posttreatment reported similar concerning results; $81 \%$ were found to have elevated total cholesterol, $15 \%$ had low HDL, and 44\% had elevated triglycerides. ${ }^{18}$

The prevalence of hypertension is similarly increased in patients with GCTs who have received prior chemotherapy, including in $25 \%$ of patients in the observational study just mentioned. ${ }^{18,38} \mathrm{~A}$ large-scale study corroborated these findings. ${ }^{38}$ In 1289 GCT survivors, the prevalence of hypertension was greater in patients treated with cisplatin compared with those treated with surgery alone, and the rates increased with higher cumulative cisplatin doses (39\% with surgery alone vs. $50 \%$ with cisplatin of $\leq 850 \mathrm{mg}$, vs. $54 \%$ with cisplatin of $>850 \mathrm{mg} ; \mathrm{P}<.001) .{ }^{38}$ These findings remained significant after adjusting for testosterone levels and body mass index (BMI). Obesity has also been reported as increased in GCT survivors treated with chemotherapy. With a cutoff BMI of 25 or more, the previously mentioned study found $48 \%$ of GCT survivors who previously received cisplatin-based therapy were obese at a median of 15 years posttreatment. ${ }^{18}$ Like hypertension, this finding may also correlate with the cumulative dose of cisplatin administered. For instance, Sagstuen et al. ${ }^{38}$ observed an increased prevalence of BMI of 30 or greater in patients with GCTs who received greater than $850 \mathrm{mg}$ of cisplatin compared with patients treated with surgery alone (odds ratio [OR], 2.4; 95\% CI, 1.3-4.4). In contrast, patients treated with lower doses of cisplatin had only a nonsignificant trend toward an increased prevalence of obesity compared with patients treated with surgery only. 

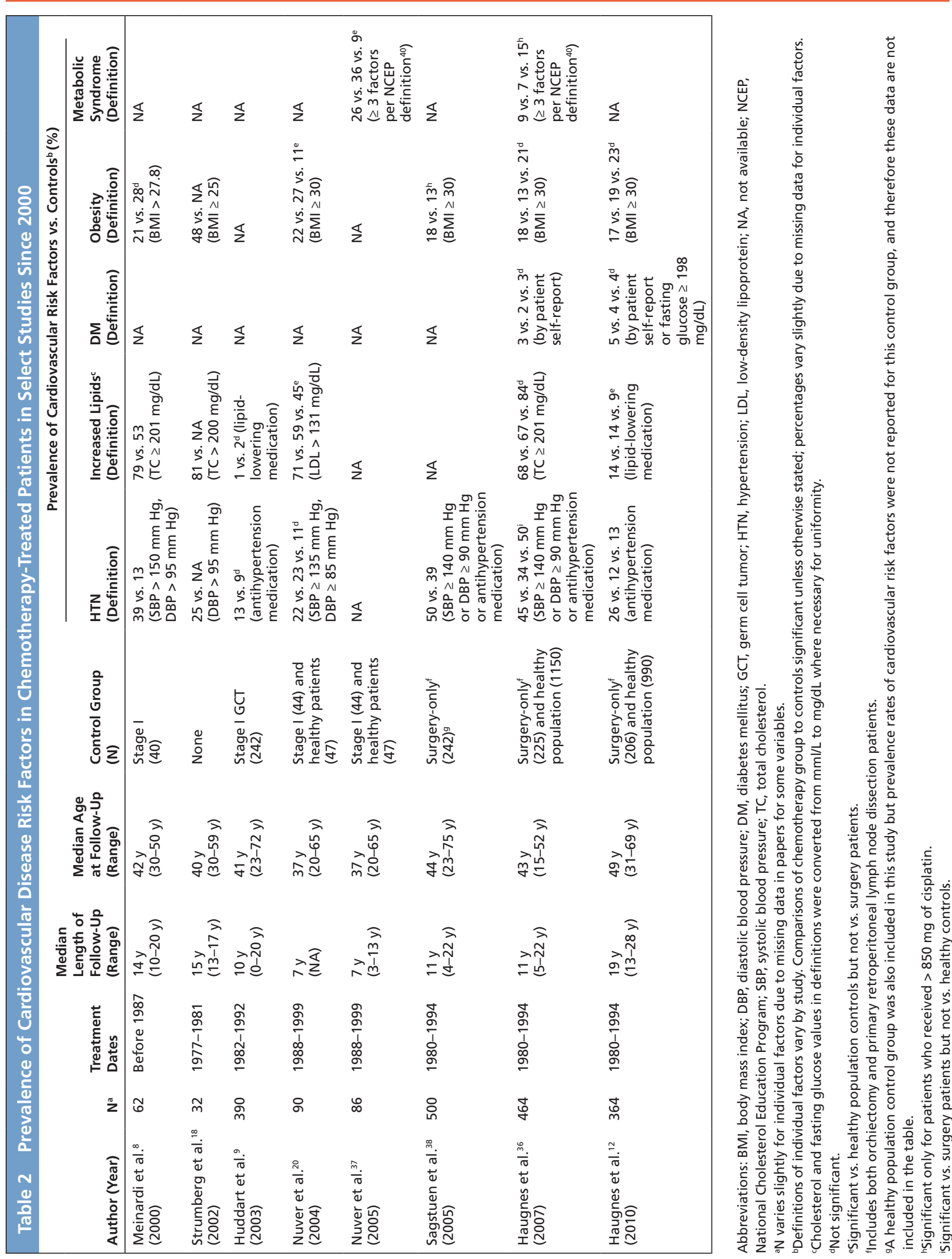
Feldman et al.

The metabolic syndrome was recently recognized as an important cardiovascular disease risk factor. ${ }^{39}$ Using a modified definition, the metabolic syndrome was reported in up to $40 \%$ of chemotherapy-treated GCT survivors younger than 60 years at a median of 11 years posttreatment. ${ }^{36}$ This rate was significantly higher than that for survivors treated with surgery alone, with the greatest risk incurred by those treated with more than $850 \mathrm{mg}$ of cisplatin (OR, 2.8; 95\% CI, 1.6-4.7). ${ }^{36}$ In a smaller study that used the conventional National Cholesterol Education Program Adult Treatment Panel III definition of the metabolic syndrome, ${ }^{40}$ its prevalence was increased among a combined group of patients with stage I testicular cancer and chemotherapy-treated survivors compared with controls (chemotherapy-treated, $36 \%$ vs. stage I, $25 \%$ vs. controls, $9 \%$; $P=.002) .{ }^{37} \mathrm{How}-$ ever, differences in metabolic syndrome rates were not significantly different between chemotherapy-treated patients and those managed with orchiectomy alone $(P=.232) \cdot{ }^{37}$

These studies suggest that the prevalence of major cardiac risk factors in these GCT survivors exceeds those of the general population (Table 2). Several of these comorbidities, particularly the metabolic syndrome, have been linked to testosterone deficiency, providing a possible association with testicular cancer treatment. ${ }^{37}$ However, most patients developing these risk factors have normal serum testosterone levels. ${ }^{36}$ Independent of the metabolic syndrome, decreased testosterone levels can lead to endothelial dysfunction, impaired vascular smooth muscle reactivity, increased intima and media thickness of vessels, and increased synthesis of proinflammatory cytokines, as extensively reviewed by Traish et al. ${ }^{41}$ These pathologic changes offer an additional explanation for the associations between testosterone deficiency and cardiovascular events.

\section{Recommendations}

Regardless of the mechanisms contributing to the increased cardiac morbidity and mortality in this population, awareness of the increased risk offers a prime opportunity for intervention and prevention of late cardiovascular disease. Cardiac workup (e.g., referral to cardiology, electrocardiogram) in these patients should be based on clinical presentation, with an appreciation of their increased risk. With respect to cardiac risk factors, the differences in definitions, varia- tions in patient populations (including prior treatment intensity and regimen), and wide ranges of prevalence rates across the available studies (Table 2 ) and uncertainty regarding the extent those prevalence rates are increased relative to the general population make it difficult to offer informed screening recommendations beyond the accepted guidelines for primary prevention of heart disease, although these have been suggested..$^{42}$ Research is urgently needed to better define the prevalence of major cardiac risk factors in these GCT survivors as a first step toward recommending heightened screening and earlier intervention.

In the interim, until these data are available, patients undergoing treatment should be made aware of their future risk for these comorbidities and encouraged to establish care with an internist familiar with their increased likelihood of developing cardiovascular disease. Lifestyle changes should be highly encouraged. Patients should be given recommendations for adopting a heart-healthy diet and regular exercise as per the American Heart Association guidelines and, if necessary, to lose weight to achieve a goal BMI less than 25. Referral to a smoking cessation program (if applicable) is also critical, because up to $40 \%$ of patients with GCTs who were smokers at diagnosis may continue smoking for many years after treatment. ${ }^{43}$ In addition, early institution of medications to more tightly regulate lipids and blood pressure may be beneficial. Primary prevention of coronary artery disease with aspirin should be considered in the context of each patient's risk. Administration of testosterone replacement to hypogonadal patients, particularly those with the metabolic syndrome, should also be considered.

Studies testing interventions are also greatly needed to provide evidence-based guidelines specifically addressing the increased cardiovascular risk in GCT survivors. In addition, physicians should have a low threshold for assessing GCT survivors with symptoms of angina for coronary artery disease or an acute coronary syndrome, regardless of age. Genetic factors, such as polymorphisms in the androgen receptor gene, leptin receptor gene, $9 \mathrm{p} 21$ (for coronary artery disease), or genes increasing the risks of diabetes and hyperlipidemia, may help determine which patients will develop cardiovascular disease with treatment. Further understanding of the role of genetics may allow for interventions specifically targeting GCT survivors at highest risk. 
Chemotherapy-Induced Cardiovascular Toxicity

\section{Conclusions}

Surviving cancer, particularly metastatic disease, is an enormous accomplishment that requires courage, perseverance, quality medical care, a good support system, and even luck. It is concerning that the survival benefit of modern treatment of GCTs may be compromised by the consequences of treatment, particularly cardiovascular morbidity. Efforts are needed to better understand the mechanisms of treatment-related cardiac toxicities so that preventive and therapeutic interventions can be instituted. Since 1981, GCTs have been regarded as "a model for the curable cancer," ${ }^{3}$ with the paradigms for their treatment extrapolated to the management of other neoplasms. Now, GCTs are again poised to help advance oncologic care, but this time in understanding and improving cancer survivorship. In doing so, GCTs have the opportunity to serve not only as "a model for the curable cancer" but also as "a model for the cured patient."

\section{References}

1. Jemal A, Siegel R, Ward E, et al. Cancer statistics, 2009. CA Cancer J Clin 2009;59:225-249.

2. Horner MJ, Ries LA, Krapcho M, et al, eds. SEER Cancer Statistics Review, 1975-2006, National Cancer Institute. Bethesda, MD. Available at: http://seer.cancer.gov/csr/1975_2006/. Accessed February 6, 2012.

3. Einhorn LH. Testicular cancer as a model for a curable neoplasm: The Richard and Hinda Rosenthal Foundation Award lecture. Cancer Res 1981;41:3275-3280.

4. Feldman DR, Bosl GJ, Sheinfeld J, et al. Medical treatment of advanced testicular cancer. JAMA 2008;299:672-684.

5. Efstathiou E, Logothetis CJ. Review of late complications of treatment and late relapse in testicular cancer. J Natl Compr Canc Netw 2006;4:1059-1070.

6. Einhorn LH, Donohue J. Cis-diamminedichloroplatinum, vinblastine, and bleomycin combination chemotherapy in disseminated testicular cancer. Ann Intern Med 1977;87:293-298.

7. Edwards GS, Lane M, Smith FE. Long-term treatment with cisdichlorodiammineplatinum(II)-vinblastine-bleomycin: possible association with severe coronary artery disease. Cancer Treat Rep 1979;63:551-552.

8. Meinardi MT, Gietema JA, van der Graaf WT, et al. Cardiovascular morbidity in long-term survivors of metastatic testicular cancer. J Clin Oncol 2000;18:1725-1732.

9. Huddart RA, Norman A, Shahidi M, et al. Cardiovascular disease as a long-term complication of treatment for testicular cancer. J Clin Oncol 2003;21:1513-1523.

10. van den Belt-Dusebout AW, Nuver J, de Wit R, et al. Long-term risk of cardiovascular disease in 5-year survivors of testicular cancer. J Clin Oncol 2006;24:467-475.

11. van den Belt-Dusebout AW, de Wit R, Gietema JA, et al. Treatment-specific risks of second malignancies and cardiovascular disease in 5-year survivors of testicular cancer. J Clin Oncol 2007;25:4370-4378.

12. Haugnes HS, Wethal $\mathrm{T}$, Aass $\mathrm{N}$, et al. Cardiovascular risk factors and morbidity in long-term survivors of testicular cancer: a 20-year follow-up study. J Clin Oncol 2010;28:4649-4657.

13. Pliarchopoulou K, Pectasides D. Late complications of chemotherapy in testicular cancer. Cancer Treat Rev 2010;36:262267.

14. Vogelzang NJ, Bosl GJ, Johnson K, et al. Raynaud's phenomenon: a common toxicity after combination chemotherapy for testicular cancer. Ann Intern Med 1981;95:288-292.

15. Glendenning JL, Barbachano Y, Norman AR, et al. Long-term neurologic and peripheral vascular toxicity after chemotherapy treatment of testicular cancer. Cancer 2010;116:2322-2331.

16. Brydoy M, Oldenburg J, Klepp O, et al. Observational study of prevalence of long-term Raynaud-like phenomena and neurological side effects in testicular cancer survivors. J Natl Cancer Inst 2009;101:1682-1695.

17. Bokemeyer C, Berger CC, Kuczyk MA, et al. Evaluation of longterm toxicity after chemotherapy for testicular cancer. J Clin Oncol 1996;14:2923-2932.

18. Strumberg D, Brugge S, Korn MW, et al. Evaluation of long-term toxicity in patients after cisplatin-based chemotherapy for nonseminomatous testicular cancer. Ann Oncol 2002;13:229-236.

19. Bissett D, Kunkeler L, Zwanenburg L, et al. Long-term sequelae of treatment for testicular germ cell tumours. Br J Cancer 1990;62:655-659.

20. Nuver J, Smit AJ, Sleijfer DT, et al. Microalbuminuria, decreased fibrinolysis, and inflammation as early signs of atherosclerosis in long-term survivors of disseminated testicular cancer. Eur J Cancer 2004;40:701-706.

21. Hansen $S W$, Olsen N. Raynaud's phenomenon in patients treated with cisplatin, vinblastine, and bleomycin for germ cell cancer: measurement of vasoconstrictor response to cold. J Clin Oncol 1989;7:940-942.

22. Heier MS, Nilsen T, Graver V, et al. Raynaud's phenomenon after combination chemotherapy of testicular cancer, measured by laser Doppler flowmetry. A pilot study. Br J Cancer 1991;63:550-552.

23. de Wit R, Stoter G, Kaye SB, et al. Importance of bleomycin in combination chemotherapy for good-prognosis testicular nonseminoma: a randomized study of the European Organization for Research and Treatment of Cancer Genitourinary Tract Cancer Cooperative Group. J Clin Oncol 1997;15:1837-1843.

24. Dirix LY, Libura M, Libura J, et al. In vitro toxicity studies with mitomycins and bleomycin on endothelial cells. Anticancer Drugs 1997;8:859-868.

25. Shi Y, Inoue S, Shinozaki R, et al. Release of cytokines from human umbilical vein endothelial cells treated with platinum compounds in vitro. Jpn J Cancer Res 1998;89:757-767.

26. Gerstein HC, Mann JF, Yi Q, et al. Albuminuria and risk of cardiovascular events, death, and heart failure in diabetic and nondiabetic individuals. JAMA 2001;286:421-426.

27. Nuver J, Smit AJ, van der Meer J, et al. Acute chemotherapyinduced cardiovascular changes in patients with testicular cancer. J Clin Oncol 2005;23:9130-9137.

28. Vaughn DJ, Palmer SC, Carver JR, et al. Cardiovascular risk in long-term survivors of testicular cancer. Cancer 2008;112:1949_ 1953.

29. O'Leary DH, Polak JF, Kronmal RA, et al. Carotid-artery intima and media thickness as a risk factor for myocardial infarction and 
Feldman et al.

stroke in older adults. Cardiovascular Health Study Collaborative Research Group. N Engl J Med 1999;340:14-22.

30. Ramesh G, Reeves WB. TNF-alpha mediates chemokine and cytokine expression and renal injury in cisplatin nephrotoxicity. J Clin Invest 2002;110:835-842.

31. Davis CA, Nick HS, Agarwal A. Manganese superoxide dismutase attenuates cisplatin-induced renal injury: importance of superoxide. J Am Soc Nephrol 2001;12:2683-2690.

32. Togna GI, Togna AR, Franconi M, et al. Cisplatin triggers platelet activation. Thromb Res 2000;99:503-509.

33. Schilsky RL, Anderson T. Hypomagnesemia and renal magnesium wasting in patients receiving cisplatin. Ann Intern Med 1979;90:929-931.

34. Turlapaty PD, Altura BM. Magnesium deficiency produces spasms of coronary arteries: relationship to etiology of sudden death ischemic heart disease. Science 1980;208:198-200.

35. Vogelzang NJ, Torkelson JL, Kennedy BJ. Hypomagnesemia, renal dysfunction, and Raynaud's phenomenon in patients treated with cisplatin, vinblastine, and bleomycin. Cancer 1985;56:2765-2770.

36. Haugnes HS, Aass N, Fossa SD, et al. Components of the metabolic syndrome in long-term survivors of testicular cancer. Ann Oncol 2007;18:241-248.
37. Nuver J, Smit AJ, Wolffenbuttel BH, et al. The metabolic syndrome and disturbances in hormone levels in long-term survivors of disseminated testicular cancer. J Clin Oncol 2005;23:3718-3725.

38. Sagstuen H, Aass N, Fossa SD, et al. Blood pressure and body mass index in long-term survivors of testicular cancer. J Clin Oncol 2005;23:4980-4990.

39. Lakka HM, Laaksonen DE, Lakka TA, et al. The metabolic syndrome and total and cardiovascular disease mortality in middleaged men. JAMA 2002;288:2709-2716.

40. Expert Panel on Detection, Evaluation, and Treatment of High Blood Cholesterol in Adults. Executive summary of the third report of the National Cholesterol Education Program (NCEP) expert panel on detection, evaluation, and treatment of high blood cholesterol in adults (Adult Treatment Panel III). JAMA 2001;285:2486-2497.

41. Traish AM, Guay A, Feeley R, et al. The dark side of testosterone deficiency: I. Metabolic syndrome and erectile dysfunction. J Androl 2009;30:10-22.

42. Vaughn DJ, Gignac GA, Meadows AT. Long-term medical care of testicular cancer survivors. Ann Intern Med 2002;136:463-470.

43. Hentrich M, Fegg MJ, Meiler S, et al. Smoking cessation in longterm survivors of germ cell tumour. J Cancer Res Clin Oncol 2006;132:557-560 